\title{
Biological Effects of Phosphate on Fibroblast-Like Synoviocytes
}

\author{
Yubo Sun, David R. Mauerhan, Deepthi Chaturvedi, \\ Edward N. Hanley Jr., and Helen E. Gruber \\ Department of Orthopaedic Surgery, Carolinas Medical Center, P.O. Box 32681, Charlotte, \\ NC 28232, USA \\ Correspondence should be addressed to Yubo Sun, yubo.sun@carolinas.org
}

Received 17 October 2011; Accepted 10 November 2011

Academic Editor: A. Chiarini

Copyright () 2012 Yubo Sun et al. This is an open access article distributed under the Creative Commons Attribution License, which permits unrestricted use, distribution, and reproduction in any medium, provided the original work is properly cited.

This study sought to examine the expression of genes implicated in phosphate transport and pathological calcification in osteoarthritis (OA) and rheumatoid arthritis (RA) fibroblast-like synoviocytes (FLS) and investigate the biological effects of phosphate. Results revealed that several genes, which were implicated in phosphate transport and pathological calcification, were differentially expressed in OA FLS and RA FLS. Phosphate stimulated the expression of matrix metalloproteinse-1, matrix metalloproteinse-3, cyclooxygenase-2, and interleukin- $1 \beta$ in a dose-dependent manner. Phosphate also induced OA FLS cell death but not RA FLS cell death at higher concentration. Calcification inhibitors, phosphocitrate (PC), and ethane-1-hydroxy1,1-diphosphonate (EHDP), effectively inhibited these detrimental biological effects of phosphate. These findings suggest that abnormal expression of genes implicated in phosphate transport and pathological calcification may contribute to the progression of $\mathrm{OA}$ through the induction of extracellular matrix-degrading enzymes, proinflammatory cytokines, cell death, and calcium deposits. Calcification inhibitors such as PC and EHDP are potent inhibitors of these detrimental biological effects of phosphate.

\section{Introduction}

Osteoarthritis (OA) is characterized by the degeneration of articular cartilage. Although the precise biochemical events that initiates OA are not well understood, many risk or contributory factors have been identified including aging, obesity, and pathological calcification. Basic calcium phosphate (BCP) and calcium pyrophosphate dihydrate (CPPD) crystals are the two most common forms of articular calcium phosphate crystals. These crystals are found in the synovial fluid of the patients with OA, and their presence in the synovial fluid correlates with the radiographic evidence of cartilaginous degeneration [1-3]. Calcium phosphate crystals were also found in the synovial fluid of up to $26 \%$ of the patients with rheumatoid arthritis (RA), and a worse clinical outcome was associated with the presence of these crystals [4].

BCP and CPPD crystals stimulate the expression of matrix metalloproteinases (MMPs), mitogenesis, and endocytotic activity of cells in monolayer culture $[5,6]$. These crystals may also alter the biomechanical properties of menisci and articular cartilage [7]. Recently, Cheung et al. demonstrated that calcium phosphocitrate (CaPC), a potent calcification inhibitor, reduced the degeneration of articular cartilage in Hartley guinea pig [8]. Their study has provided support for the hypothesis that pathological calcification is a therapeutic target for the treatment of crystal-associated OA.

Treatment of human fibroblasts with BCP crystals induced a transient 10 -fold rise of intracellular calcium within seconds and a second sustained rise starting at 60 minutes, which resulted from crystal dissolution [9]. It is conceivable that the intracellular concentration of phosphate could also rise due to crystal dissolution. To reduce the elevated intracellular concentration of phosphate, phosphate ions transporters or other mechanisms must be activated to pump out the extra intracellular phosphate, which, in turn, may lead to a transient rise of the concentration of local extracellular phosphate.

Phosphate plays a critical role in skeletal development, mineral metabolism, and diverse cellular functions. Besides being a key component of biomineral, it is a vital component of DNA, RNA, ATP, phospholipids, and phosphorylated 
proteins. It has been shown that phosphate plays an important role in chondrocyte differentiation, chondrocyte death, and biomineralization within the growth plate cartilage [10-12]. Since $100 \%$ of OA patients have synovial crystals, phosphate ions in the synovial fluid before crystal being formed or after crystal dissolution may potentially have some effects on fibroblast-like synoviocyte (FLS). The biological effects of calcium phosphate crystals on FLS have been examined extensively $[5,6,13]$; however, the biological effect of phosphate on FLS has not been studied. We carried out this study to investigate the expression of several genes implicated in phosphate transport and pathological calcification in OA FLS and examine the biological effects of phosphate.

\section{Methods}

Dulbecco's minimum essential medium (DMEM), fetal bovine serum (FBS), and stock antibiotic/antimycotic mixture were products of Invitrogen (Carlsbad, CA, USA). OA and RA FLS cell lines, hTERT-OA 13A FLS and hTERT-RA 516 FLS, have been described previously $[14,15]$.

2.1. Cell Culture and Phosphate Treatment. hTERT-OA 13A FLS and hTERT-RA 516 FLS were plated at 90\% confluence in $100 \mathrm{~mm}$ plates. On the second day, DMEM containing $10 \%$ FBS was added. Twenty-four hours later, RNA was extracted. For phosphate treatment experiments, hTERT-OA 13A FLS and hTERT-RA 516 FLS were plated at $85 \%$ confluence in $100 \mathrm{~mm}$ plates. On the second day, DMEM containing $0.5 \%$ FBS was added. Twenty-four hours later, cells were treated with increasing amounts of $\mathrm{NaH}_{2} \mathrm{PO}_{4}$ in DMEM containing $0.5 \%$ serum. Twenty-four hours posttreatment, total RNA was extracted.

2.2. Semiquantitative RT-PCR. Briefly, $1 \mu \mathrm{g}$ of RNA sample was reverse transcribed at $50^{\circ} \mathrm{C}$ for 60 minutes, followed by enzyme inactivation at $85^{\circ} \mathrm{C}$ for 5 minutes. RT-PCR was carried out using gene-specific primers and a ThermoScript RT-PCR System (Invitrogen, Carlsbad, CA, USA). Amplifications were carried out for $28-40$ cycles by denaturing at $95^{\circ} \mathrm{C}$ for 30 seconds, annealing at $55^{\circ} \mathrm{C}$ for 30 seconds, and extending at $72^{\circ} \mathrm{C}$ for 45 seconds, with a final extension at $72^{\circ} \mathrm{C}$ for 10 minutes. PCR products were electrophoresed on $2 \%$ agarose gels, stained with ethidium bromide, and photographed using a low light image system (ChemiImager 4000, Alpha Innotech Corporation, San Leandro, CA, USA). Each RT-PCR experiment was repeated twice using two different batches of RNA samples.

2.3. Cell Morphology and Viability. The effects of phosphate on cell morphology and viability were examined. Briefly, hTERT-OA 13A FLS and hTERT-RA 516 FLS were plated in six-well plates at a density of $1.8 \times 10^{5} /$ well. Twenty-four hours later, the media were changed to DMEM media with $0.5 \%$ FBS and cultured for forty-eight hours. The serumstarved cells were then treated with increasing amounts of $\mathrm{NaH}_{2} \mathrm{PO}_{4}$ in serum-free DMEM. Twenty-four hours later, the cells were examined and photographed under a microscope. The cells were then harvested, and number of cells was counted using a hemocytometer.

2.4. Inhibition by $P C$. To investigate the inhibitory activity of calcification inhibitors on the biological effects of phosphate, hTERT-OA 13A FLS were treated with $\mathrm{NaH}_{2} \mathrm{PO}_{4}$ at the presence or absence of PC or EHDP at a final concentration of $20 \mu \mathrm{M}$.

\section{Results}

3.1. Expression of Genes Implicated in Phosphate Transport and Pathological Calcification. We have recently carried out a microarray analysis of hTERT-OA 13A FLS and hTERTRA 516 FLS and found that several genes implicated in lipid transfer, phosphate transport, or pathological calcification were differentially expressed in OA and RA FLS cell lines hTERT-OA 13A FLS and hTERT-RA 516 FLS [16]. These genes included macrophage scavenger receptor type I (MSR1), scavenger receptor class A, member 5 (SCARA5), progressive ankylosis homolog (ANKH), ectonucleotide pyrophosphatase-phosphodiesterase 2 (ENPP2), adenylyl cyclase 3 (ADCY3), and ectonucleoside triphosphate diphosphohydrolase 1 (ENTPD1). To confirm these differential expressions, we decided to examine the expression of these genes using semiquantitative RT-PCR. Besides these genes, we also examined the expression of ectonucleotide pyrophosphatase-phosphodiesterase 1 (ENPP1) and tissue nonspecific alkaline phosphatase (TNSAP) using semiquantitative RT-PCR.

MSR1 and SCARA5 are involved in both lipid and phosphate transport $[17,18]$. ANKH is a multipass transmembrane protein, which regulates transport of pyrophosphate ions across the plasma membrane [19]. ENTPD1 is an integral membrane ectoapyrase that hydrolyzes extracellular ATP/ADP. ADCY3 is a membrane-associated enzyme that converts ATP to cAMP. ENPP1 and ENPP2 are phosphodiesterase and nucleotide pyrophosphatase that hydrolyzes nucleotides to pyrophosphate/phosphate $[20,21]$. TNSAP is a pyrophosphatase that hydrolyzes pyrophosphate. ENPP1, TNSAP, and ANKH have been previously implicated in pathological calcification in OA [22-25].

As shown in Figure 1, the expression of MSR1, SCARA5, ENTPD1, ENPP1, ENPP2, TNSAP, and ADCY3 were upregulated in hTERT-OA 13A FLS compared to hTERT-RA 516 FLS. These results are consistent with the previous findings that elevated activity of ENPP1 and TNSAP was detected in OA cartilage and OA-derived chondrocytes [22-24].

3.2. Effects of Phosphate on the Expressions of $M M P s, I L-1 \beta$, and Cox-2. To investigate the biological effect of phosphate and its potential role in disease progression of $\mathrm{OA}$, we treated hTERT-OA 13A FLS with increasing amounts of $\mathrm{NaH}_{2} \mathrm{PO}_{4}$ and analyzed the expression of matrix metalloproteinase-1 (MMP1), matrix metalloproteinase-3 (MMP3), and interleukin-1 $\beta$ (IL-1 $\beta)$. As shown in Figure $2(\mathrm{a}), \mathrm{NaH}_{2} \mathrm{PO}_{4}$, similar to BCP crystals $[5,26,27]$, stimulated the expression 

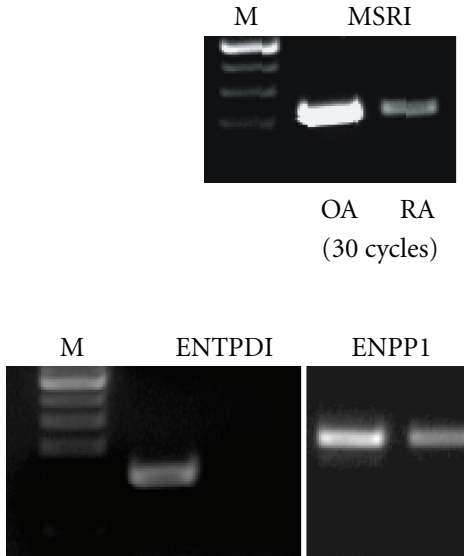

OA RA

(35 cycles)
OA RA

(30 cycles)

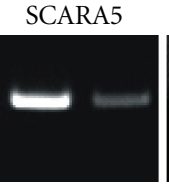

OA RA

(40 cycles)

(a)

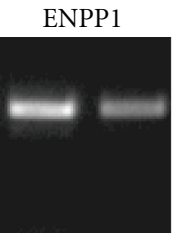

OA RA

(35 cycles)

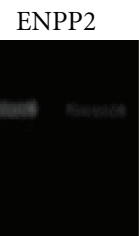

OA RA

(35 cycles)

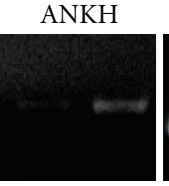

OA RA

(40 cycles)

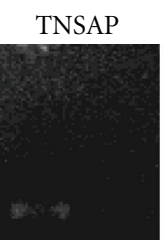

OA RA

(35 cycles)

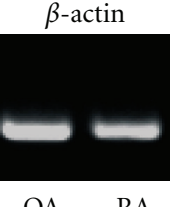

(30 cycles)

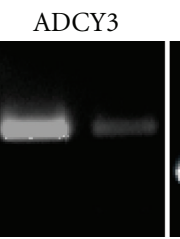

OA RA

(40 cycles)

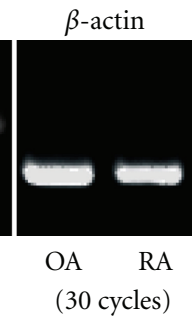

(b)

FIGURE 1: Differential expressions of genes implicated in lipid transfer, phosphate transport, and pathological calcification. hTERT-OA 13A FLS and hTERT-RA 516 FLS were plated in $100 \mathrm{~mm}$ plates at $90 \%$ confluence. On the second day, medium containing $10 \%$ serum was added. Twenty-four hours later, RNA was extracted and examined with semiquantitative RT-PCR for the expression of MSR1, SCARA5, ANKH, ENTPD1, ENPP1, ENPP2, TNSAP, and ADCY3.
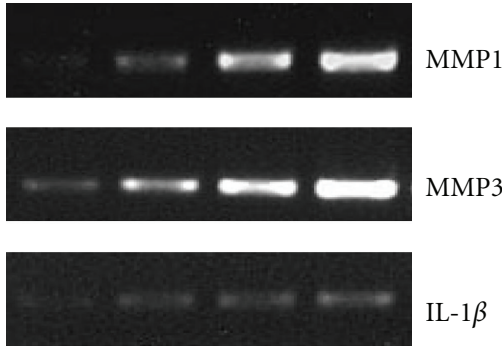

MMP3
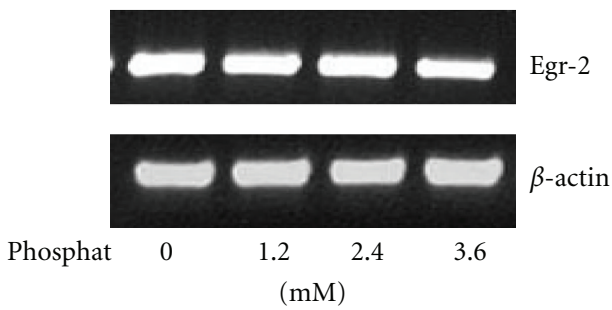

(a)
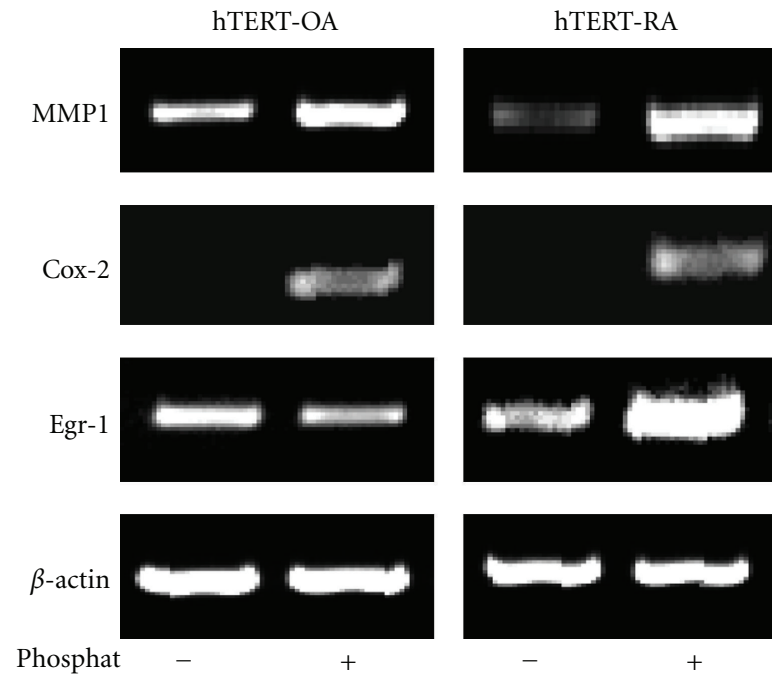

(b)

FIGURE 2: The effect of phosphate on the expression of MMPs, IL-1 $\beta$, and Cox-2. (a) hTERT-OA 13A FLS were treated with increasing amounts of $\mathrm{NaH}_{2} \mathrm{PO}_{4}$. The expression of MMP1, MMP3, IL-1 $\beta$, and egr-2 was examined. (b) hTERT-OA 13A FLS and hTERT-RA 516 FLS were treated with $\mathrm{NaH}_{2} \mathrm{PO}_{4}$ at a final concentration of $2.6 \mathrm{mM}$ for twenty-four hours. The expression of MMP1, Cox-2, and egr-1 was examined.

of MMP1, MMP3, and IL-1 $\beta$ in a dose-dependent manner. However, unlike BCP crystals [28], $\mathrm{NaH}_{2} \mathrm{PO}_{4}$ had no effect on the expression of early growth response-2 (egr-2). We also examined the expression of MMP1, cyclooxygenase-2 (Cox2 ), and early growth response-1 (egr-1) in both hTERT-OA 13A FLS and hTERT-RA 516 FLS. As shown in Figure 2(b), $\mathrm{NaH}_{2} \mathrm{PO}_{4}$ stimulated the expression of MMP1 and Cox-2 in both hTERT-OA 13A FLS and hTERT-RA 516 FLS. Interestingly, $\mathrm{NaH}_{2} \mathrm{PO}_{4}$ stimulated the expression of egr-1 in hTERT-RA 516 FLS but suppressed it in hTERT-OA 13A FLS.

3.3. Phosphate Induces Cell Death of OA FLS. It has been shown that phosphate plays an important role in chondrocyte differentiation and death at a higher concentration [10-12]. Therefore, we decided to examine the effect of phosphate on FLS at a higher concentration. As shown in Figure 3, $\mathrm{NaH}_{2} \mathrm{PO}_{4}$ treatment at a concentration $3.2 \mathrm{mM}$ 
TABLE 1: Cell number.

\begin{tabular}{lcccc}
\hline Phosphate $(\mathrm{mM})$ & 0 & 1 & 2 & 3 \\
\hline hTERT-OA 13A FLS & $1.8 \times 10^{5}$ & $1.3 \times 10^{5}$ & $0.9 \times 10^{5}$ & $0.3 \times 10^{5}$ \\
hTERT-RA 516 FLS & $1.8 \times 10^{5}$ & $1.9 \times 10^{5}$ & $1.9 \times 10^{5}$ & $1.6 \times 10^{5}$ \\
\hline
\end{tabular}

hTERT-OA 13A FLS and hTERT-RA 516 FLS were plated in six-well plates at a density of $1.8 \times 10^{5} /$ well. Twenty-four hours later, the media were changed to serum-free DMEM media and cultured for forty-hours. The cells were then treated with increasing amounts of $\mathrm{NaH}_{2} \mathrm{PO}_{4}$. Twenty-four hours later, the cells were harvested, and cells numbers were counted using a hemocytometer.

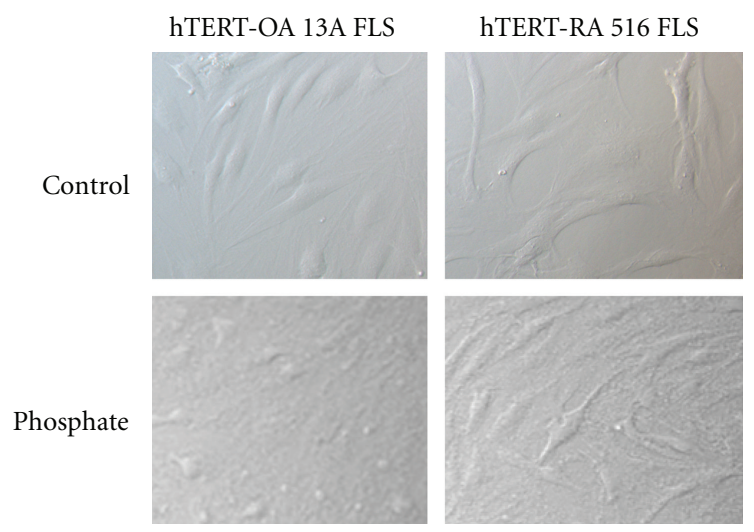

Figure 3: Phosphate induces cell death of hTERT-OA 13A FLS. Serum-starved hTERT-OA 13A FLS and hTERT-RA 516 FLS were treated with $\mathrm{NaH}_{2} \mathrm{PO}_{4}$ at a final concentration of $3.2 \mathrm{mM}$ for twenty-four hours. The cells were photographed under microscope. Results demonstrated that $\mathrm{NaH}_{2} \mathrm{PO}_{4}$ at a final concentration of $3.2 \mathrm{mM}$ caused cell shrinkage and induced cell death of hTERTOA 13A FLS but had less effect on the morphology and viability of hTERT-RA 516 FLS.

caused severe cell shrinkage and cell death in serum-starved hTERT-OA 13A FLS. More than $80 \%$ of the phosphatetreated hTERT-OA 13A FLS were shrunken or dead after a twenty-four-hour phosphate treatment. Interestingly, the same treatment had much less effect on the viability of hTERT-RA 516 FLS (Figure 3). It is worth noting that calcium phosphate deposits were formed and deposited on these FLS cells, especially on the OA FLS.

To further investigate the effect of phosphate on the cell viability, we treated serum-starved hTERT-OA 13A FLS and hTERT-RA 516 with increasing amounts of $\mathrm{NaH}_{2} \mathrm{PO}_{4}$. Twenty-four hours later, the numbers of cells were determined by cell count using a hemocytometer. As shown in Table 1, phosphate reduced the viability of hTERT-OA 13A FLS in a dose-dependent manner, while had no significant effect on the viability of hTERT-RA 516 FLS.

\subsection{PC and EHDP Inhibited the Detrimental Biological Effects} of Phosphate. We have previously shown that calcification inhibitors PC and ethane-1-hydroxy-1,1-diphosphonate, (EHDP) inhibit crystals-induced endocytotic activity and calcium-phosphate-DNA aggregates induced cell death $[6$, 29]. We thought that the inhibitors might also inhibit the detrimental biological effects of phosphate. To test this, we treated hTERT-OA 13A FLS with $\mathrm{NaH}_{2} \mathrm{PO}_{4}$ at the presence or absence of PC or EHDP. As shown in Figure 4, PC and
EHDP effectively inhibited phosphate-induced cell death and the expression of MMP1 and IL- $1 \beta$.

\section{Discussion}

Studies have found that excess intake of phosphate for long periods is an important factor in bone impairment and aging [30]. In addition, elevation of extracellular phosphate increases the production of reactive oxygen species [31]. In the present study, we demonstrate that several genes implicated in phosphate transport and pathological calcifications are differentially expressed in OA and RA FLS cell lines hTERT-OA 13A FLS and hTERT-RA 516 FLS. Significantly, TNSAP and ENPP1 are expressed at higher levels in hTERTOA FLS compared to hTERT-RA 516 FLS. TNSAP and ENPP1 are known for their role in pathological calcification. The differential expression of these genes suggests that OA FLS may not be passive bystanders in OA.

Both MMPs and some proinflammatory cytokines have been implicated in OA. The finding that phosphate stimulated the expression of MMP1, MMP3, IL-1 $\beta$, and Cox-2 suggests that phosphate, similar to calcium phosphate crystals, may play a role in the progression of OA. Previous studies found that calcium phosphate crystals stimulated the expression of egr- 1 and egr-2 $[28,32]$. In this study, we found that phosphate had no effect on the expression of egr-2 and suppressed the expression of egr-1 in hTERT-OA 13A FLS. In contrast, phosphate stimulated the expression of egr-1 in hTERT-RA 516 FLS. We also found that phosphate induced cell death of hTERT-OA 13A FLS but not hTERT-RA 516 FLS. These findings are consistent with a previous report that egr-1 was expressed at lower level in OA articular cartilage [33] and that RA synovium displays severe synovial hyperplasia.

PC inhibits crystals-stimulated production of MMPs and the development of OA in Hartley guinea pigs $[8,34]$. In this study, we demonstrate that PC is a potent inhibitor of phosphate-mediated induction of MMPs and IL-1 $\beta$. PC at a final concentration $20 \mu \mathrm{M}$, which was fivefold less than the concentration of PC required to inhibit crystal-mediated induction of MMPs [29], inhibited phosphate-mediated induction of MMPs completely. This finding indicates that phosphate, similar to crystals, is a molecular target of PC.

We also demonstrate that EHDP, a stable analogue of pyrophosphate, inhibits the detrimental biological effect of phosphate. This observation suggests that pyrophosphate, an endogenous calcification inhibitor, may protect articular from degeneration. Taken together, our findings provide a support for the hypothesis that small molecule calcification 


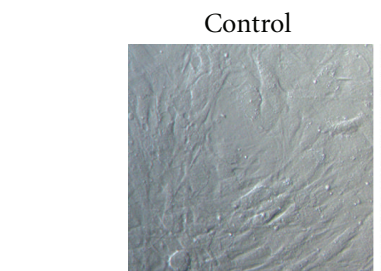

Cell number $\quad 2 \times 10^{5}$

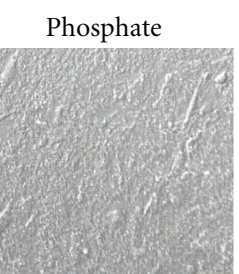

$4 \times 10^{4}$

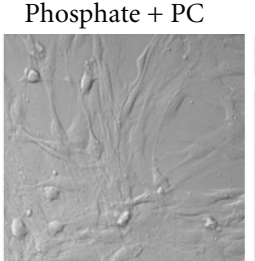

$1.7 \times 10^{5}$

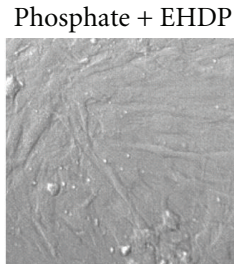

$1.4 \times 10^{5}$

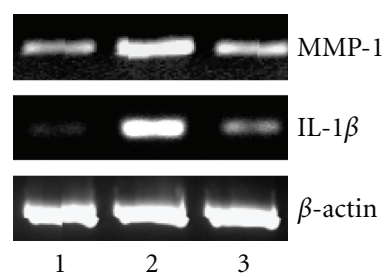

(b)

(a)

FIGURE 4: PC and EHDP inhibit the detrimental biological effects of phosphate. (a) Serum-starved hTERT-OA 13A FLS were treated with $\mathrm{NaH}_{2} \mathrm{PO}_{4}$ at a final concentration of $3.2 \mathrm{mM}$ for twenty-four hours at the presence or absence of $20 \mu \mathrm{M}$ of PC or $20 \mu \mathrm{M}$ of EHDP. PC and EHDP inhibited cell death induced by phosphate. (b) hTERT-OA 13A FLS were treated with $\mathrm{NaH}_{2} \mathrm{PO}_{4}$ at a final concentration of 2.6 mM for twenty-four hours at the presence or absence of $20 \mu \mathrm{M}$ of PC. The expression of MMP1 and IL- $1 \beta$ was examined with semiquantitative RT-PCR.

inhibitors are potential disease-modifying drugs for OA therapy.

Our study had limitations. Although phosphate-containing crystals exist in the joint fluid of patients with OA, the phosphate level in the synovial fluid may change during disease process due to disease flare, new crystal formation, and crystal dissolution. Therefore, the actual biological effect of phosphate in clinical setting requires further study. Hartley guinea pigs subjecting to a high-phosphate diet may be a suitable model for the study of the in vivo effect of phosphate and its role in the disease progression of OA.

\section{References}

[1] G. J. Carroll, R. A. Stuart, J. A. Armstrong, P. D. Breidahl, and B. A. Laing, "Hydroxyapatite crystals are a frequent finding in osteoarthritic synovial fluid, but are not related to increased concentrations of keratan sulfate or interleukin $1 \beta$," Journal of Rheumatology, vol. 18, no. 6, pp. 861-866, 1991.

[2] B. A. Derfus, J. B. Kurian, J. J. Butler et al., "The high prevalence of pathologic calcium crystals in pre-operative knees," Journal of Rheumatology, vol. 29, no. 3, pp. 570-574, 2002.

[3] S. Nalbant, J. A. M. Martinez, T. Kitumnuaypong, G. Clayburne, M. Sieck, and H. R. Schumacher Jr, "Synovial fluid features and their relations to osteoarthritis severity: new findings from sequential studies," Osteoarthritis and Cartilage, vol. 11, no. 1, pp. 50-54, 2003.

[4] J. C. Gerster, P. A. Varisco, J. Kern, J. Dudler, and A. K. L. So, "CPPD crystal deposition disease in patients with rheumatoid arthritis," Clinical Rheumatology, vol. 25, no. 4, pp. 468-469, 2006.

[5] G. M. McCarthy, P. G. Mitchell, and H. S. Cheung, "The mitogenic response to stimulation with basic calcium phosphate crystals is accompanied by induction and secretion of collagenase in human fibroblasts," Arthritis and Rheumatism, vol. 34, no. 8, pp. 1021-1030, 1991.

[6] Y. Sun, X. R. Zeng, L. Wenger, and H. S. Cheung, "Basic calcium phosphate crystals stimulate the endocytotic activity of cells-inhibition by anti-calcification agents," Biochemical and Biophysical Research Communications, vol. 312, no. 4, pp. 1053-1059, 2003.

[7] Y. Sun, D. R. Mauerhan, P. R. Honeycutt et al., "Calcium deposition in osteoarthritic menisci and meniscal cell culture," Arthritis Research \& Therapy, vol. 12, no. 2, p. R56, 2010.
[8] H. S. Cheung, J. D. Sallis, K. D. Demadis, and A. Wierzbicki, "Phosphocitrate blocks calcification-induced articular joint degeneration in a guinea pig model," Arthritis and Rheumatism, vol. 54, no. 8, pp. 2452-2461, 2006.

[9] P. B. Halverson, A. Greene, and H. S. Cheung, "Intracellular calcium responses to basic calcium phosphate crystals in fibroblasts," Osteoarthritis and Cartilage, vol. 6, no. 5, pp. 324329, 1998.

[10] D. Wang, L. Canaff, D. Davidson et al., "Alterations in the sensing and transport of phosphate and calcium by differentiating chondrocytes," Journal of Biological Chemistry, vol. 276, no. 36, pp. 33995-34005, 2001.

[11] K. Mansfield, R. Rajpurohit, and I. M. Shapiro, "Extracellular phosphate lions cause apoptosis of terminally differentiated epiphyseal chondrocytes," Journal of Cellular Physiology, vol. 179, no. 3, pp. 276-286, 1999.

[12] D. Magne, G. Bluteau, C. Faucheux et al., "Phosphate is a specific signal for ATDC5 chondrocyte maturation and apoptosisassociated mineralization: possible implication of apoptosis in the regulation of endochondral ossification," Journal of Bone and Mineral Research, vol. 18, no. 8, pp. 1430-1442, 2003.

[13] H. S. Cheung and D. J. McCarty, "Mechanisms of connective tissue damage by crystals containing calcium," Rheumatic Disease Clinics of North America, vol. 14, no. 2, pp. 365-376, 1988.

[14] Y. Sun, G. S. Firestein, L. Wenger, C. Y. C. Huang, and H. S. Cheung, "Telomerase-transduced osteoarthritic fibroblastlike synoviocyte cell line," Biochemical and Biophysical Research Communications, vol. 323, no. 4, pp. 1287-1292, 2004.

[15] Y. Sun, G. Firestein, D. Boyle, H. Gruber, and H. Cheung, "Longterm culture of telomerase-transduced rheumatoid arthritis fibroblast-like synoviocytes display a distinct gene expression pattern," Journal of Rheumatology, vol. 34, no. 10, pp. 1959-1970, 2007.

[16] Y. Sun, D. R. Mauerhan, G. S. Firestein, B. J. Loeffler, E. N. Hanley, and H. E. Gruber, "Telomerase transduced osteoarthritis fibroblast-like synoviocytes display a distinct gene expression profile," Journal of Rheumatology, vol. 36, no. 1, pp. 141-155, 2009.

[17] V. V. Kunjathoor, M. Febbraio, E. A. Podrez et al., "Scavenger receptors class A-I/II and CD36 are the principal receptors responsible for the uptake of modified low density lipoprotein leading to lipid loading in macrophages," Journal of Biological Chemistry, vol. 277, no. 51, pp. 49982-49988, 2002.

[18] K. Takahashi, M. Takeya, and N. Sakashita, "Multifunctional roles of macrophages in the development and progression of 
atherosclerosis in humans and experimental animals," Medical Electron Microscopy, vol. 35, no. 4, pp. 179-203, 2002.

[19] C. J. Williams, A. Pendleton, G. Bonavita et al., "Mutations in the amino terminus of ANKH in two US families with calcium pyrophosphate dihydrate crystal deposition disease," Arthritis and Rheumatism, vol. 48, no. 9, pp. 2627-2631, 2003.

[20] L. Hessle, K. A. Johnson, H. C. Anderson et al., "Tissuenonspecific alkaline phosphatase and plasma cell membrane glycoprotein-1 are central antagonistic regulators of bone mineralization," Proceedings of the National Academy of Sciences of the United States of America, vol. 99, no. 14, pp. 94459449, 2002.

[21] D. Harmey, L. Hessle, S. Narisawa, K. A. Johnson, R. Terkeltaub, and J. L. Millán, "Concerted regulation of inorganic pyrophosphate and osteopontin by Akp2, Enpp1, and Ank: an integrated model of the pathogenesis of mineralization disorders," American Journal of Pathology, vol. 164, no. 4, pp. 1199-1209, 2004.

[22] K. Johnson, K. Pritzker, J. Goding, and R. Terkeltaub, “The nucleoside triphosphate pyrophosphohydrolase isozyme PC-1 directly promotes cartilage calcification through chondrocyte apoptosis and increased calcium precipitation by mineralizing vesicles," Journal of Rheumatology, vol. 28, no. 12, pp. 26812691, 2001.

[23] K. Johnson, S. Hashimoto, M. Lotz, K. Pritzker, J. Goding, and R. Terkeltaub, "Up-regulated expression of the phosphodiesterase nucleotide pyrophosphatase family member PC-1 is a marker and pathogenic factor for knee meniscal cartilage matrix calcification," Arthritis and Rheumatism, vol. 44, no. 5, pp. 1071-1081, 2001.

[24] L. M. Ryan and A. K. Rosenthal, "Metabolism of extracellular pyrophosphate," Current Opinion in Rheumatology, vol. 15, no. 3, pp. 311-314, 2003.

[25] H. C. Anderson, J. B. Sipe, L. Hessle, R. Dhanyamraju, E. Atti, and N. P. Camacho, "Impaired calcification around matrix vesicles of growth plate and bone in alkaline phosphatasedeficient mice.[erratum appears in Am J Pathol. 12004 May;164(5):873 Note: Dhamyamraju, Rama [corrected to Dhanyamraju, Rama]]," American Journal of Pathology, vol. 164, no. 3, pp. 841-847, 2004.

[26] G. M. McCarthy, P. R. Westfall, I. Masuda, P. A. Christopherson, H. S. Cheung, and P. G. Mitchell, "Basic calcium phosphate crystals activate human osteoarthritic synovial fibroblasts and induce matrix metalloproteinase-13 (collagenase3 ) in adult porcine articular chondrocytes," Annals of the Rheumatic Diseases, vol. 60, no. 4, pp. 399-406, 2001.

[27] M. P. Morgan, L. C. Whelan, J. D. Sallis, C. J. McCarthy, D. J. Fitzgerald, and G. M. McCarthy, "Basic calcium phosphate crystal-induced prostaglandin E2 production in human fibroblasts: role of cyclooxygenase 1, cyclooxygenase 2, and interleukin-1 $\beta$," Arthritis and Rheumatism, vol. 50, no. 5, pp. 1642-1649, 2004.

[28] X. R. Zeng, Y. Sun, L. Wenger, and H. S. Cheung, "Induction of early growth response gene Egr2 by basic calcium phosphate crystals through a calcium-dependent protein kinase Cindependent $\mathrm{p} 44 / 42$ mitogen-activated protein kinase pathway," Cells Tissues Organs, vol. 174, no. 1-2, pp. 63-72, 2003.

[29] Y. Sun, P. Reuben, L. Wenger, J. D. Sallis, K. D. Demadis, and H. S. Cheung, "Inhibition of calcium phosphate-DNA coprecipitates induced cell death by phosphocitrates," Frontiers in Bioscience, vol. 10, pp. 803-808, 2005.

[30] E. Takeda, Y. Taketani, N. Sawada, T. Sato, and H. Yamamoto, "The regulation and function of phosphate in the human body," BioFactors, vol. 21, no. 1-4, pp. 345-355, 2004.
[31] E. Takeda, Y. Taketani, K. Nashiki et al., "A novel function of phosphate-mediated intracellular signal transduction pathways," Advances in Enzyme Regulation, vol. 46, no. 1, pp. 154$161,2006$.

[32] X. R. Zeng, Y. Sun, L. Wenger, and H. S. Cheung, "Basic calcium phosphate crystal-induced Egr-1 expression stimulates mitogenesis in human fibroblasts," Biochemical and Biophysical Research Communications, vol. 330, no. 3, pp. 658-664, 2005.

[33] F. L. Wang, J. R. Connor, R. A. Dodds et al., "Differential expression of Egr-1 in osteoarthritic compared to normal adult human articular cartilage," Osteoarthritis and Cartilage, vol. 8, no. 3, pp. 161-169, 2000.

[34] D. Nair, R. P. Misra, J. D. Sallis, and H. S. Cheung, "Phosphocitrate inhibits a basic calcium phosphate and calcium pyrophosphate dihydrate crystal-induced mitogen-activated protein kinase cascade signal transduction pathway," Journal of Biological Chemistry, vol. 272, no. 30, pp. 18920-18925, 1997. 

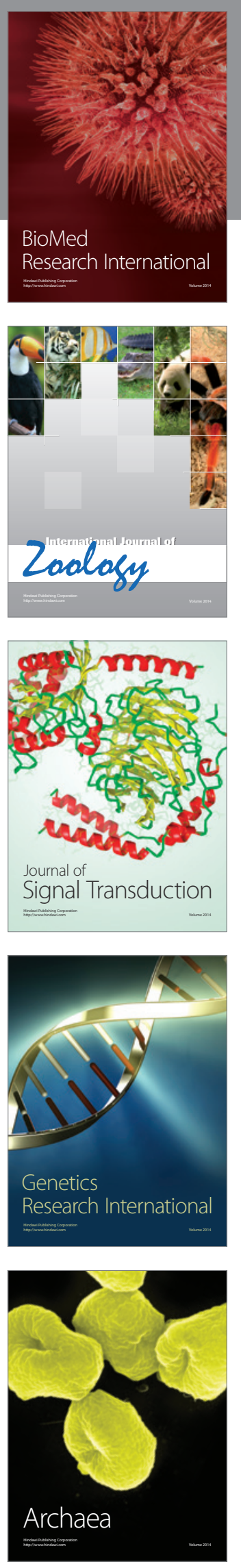
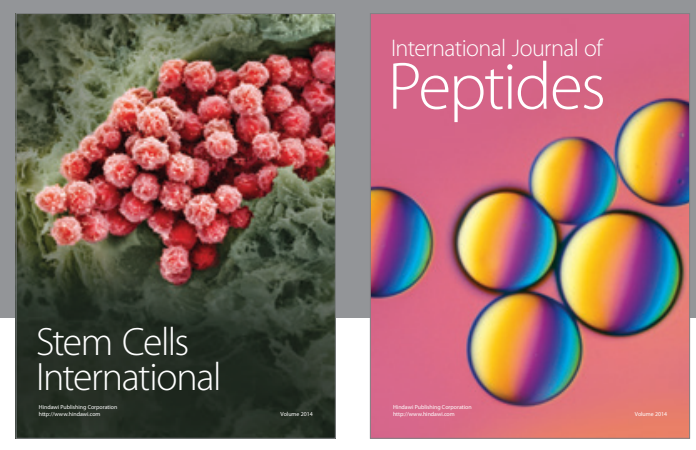

Submit your manuscripts at

http://www.hindawi.com
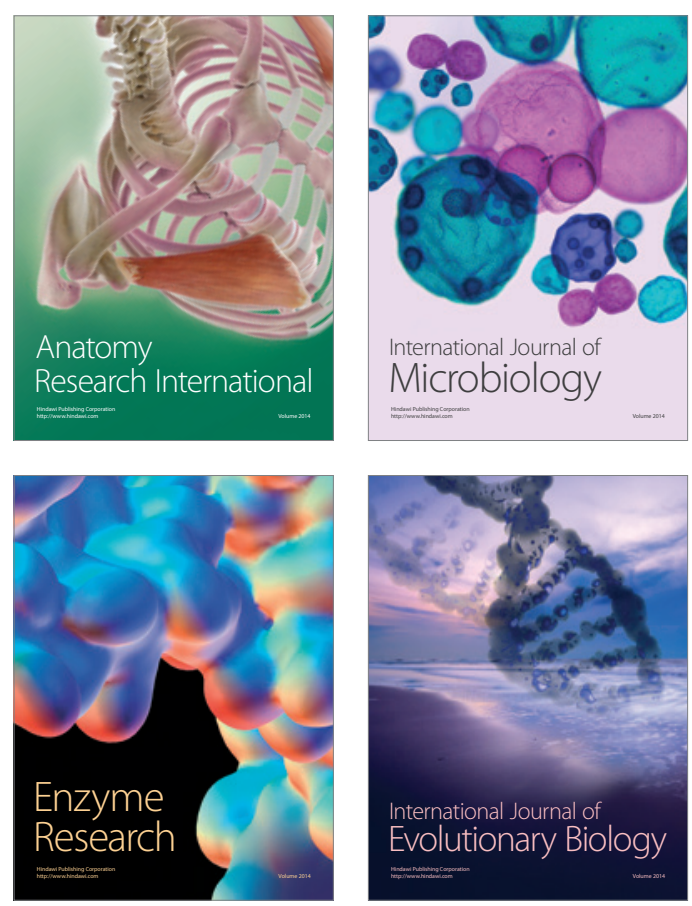
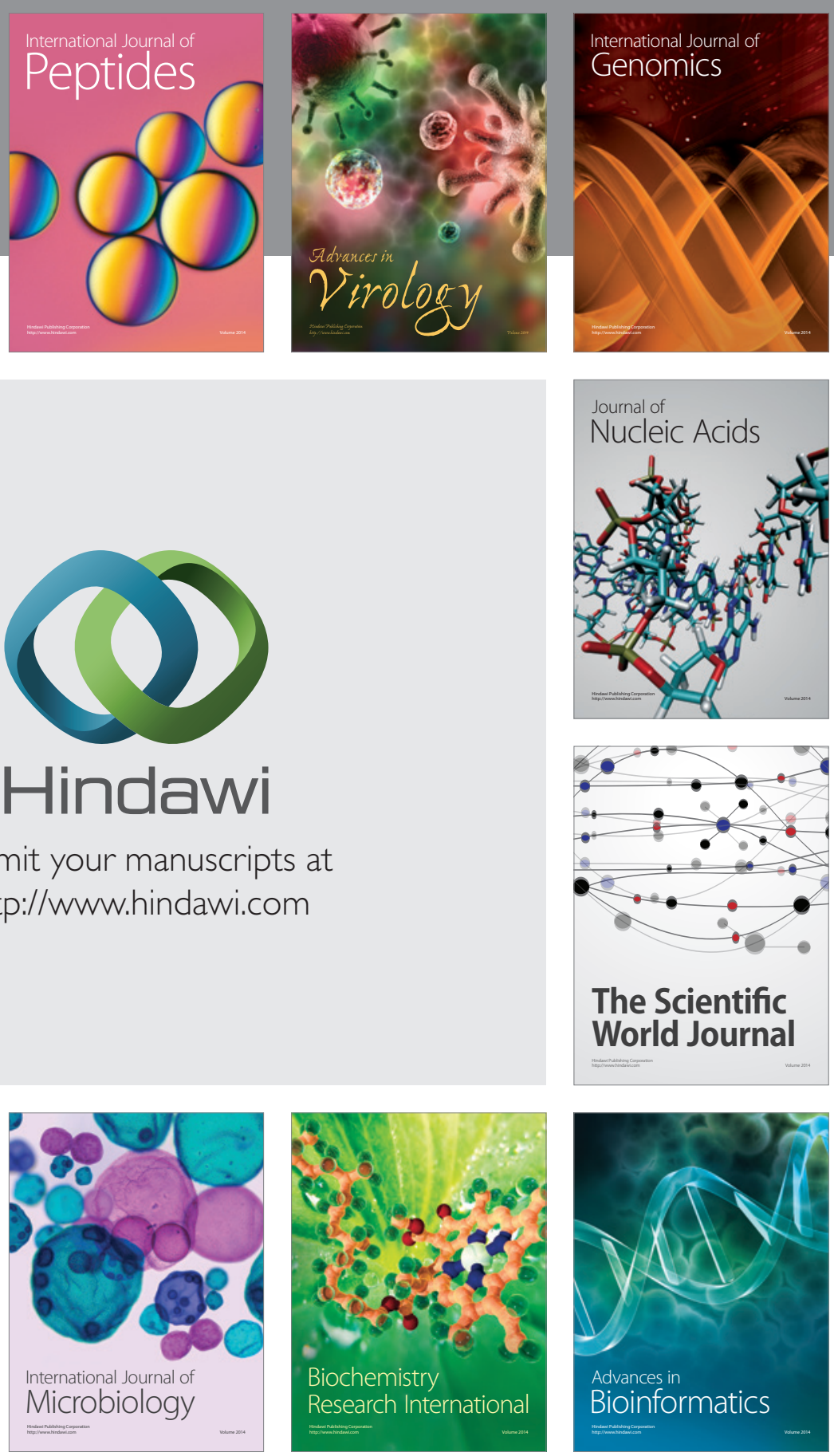

The Scientific World Journal
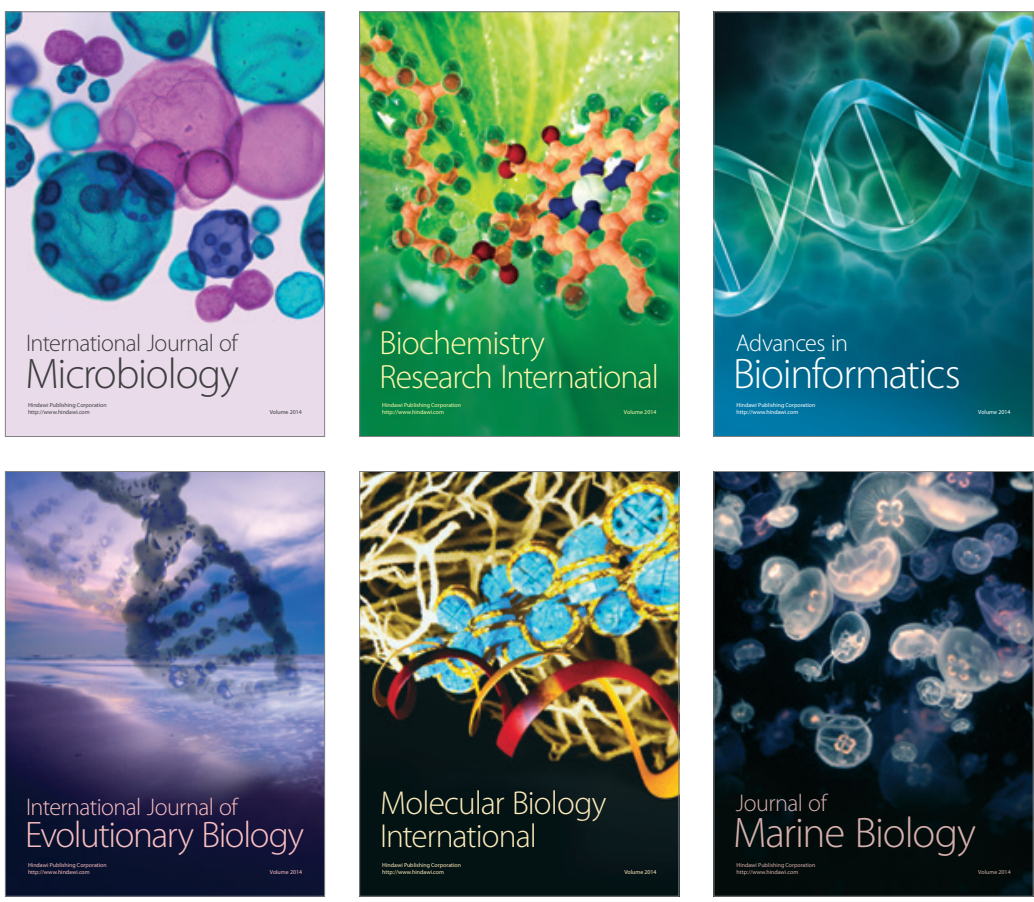